\title{
Glomus tumor in the stomach: A case report and review of the literature
}

\author{
KAI-BO CHEN and LI CHEN \\ Department of General Surgery, The Second Affiliated Hospital, College of Medicine, \\ Zhejiang University, Hangzhou, Zhejiang 310000, P.R. China
}

Received August 17, 2013; Accepted February 18, 2014

DOI: 10.3892/ol.2014.1986

\begin{abstract}
This study reports a rare case of a 47-year-old female with a gastric glomus tumor who was admitted with epigastralgia. Endoscopic ultrasound revealed a protrusion on the posterior wall of the gastric antrum. Enhanced computed tomography confirmed the presence of a $10-\mathrm{mm}$ mass. The tumor was resected, and immunohistochemistry revealed the tumor to be positive for smooth muscle actin and collagen type IV, and negative for synaptophysin, chromogranin A, laminin, S-100, cluster of differentiation (CD)34, CD31, CD99, cytokeratin (AE1/AE3), desmin and epithelial membrane antigen. The proliferation marker Ki-67 was positive in $<5 \%$ of tumor cell nuclei. The clinical procedures with a review of the literature are reported.
\end{abstract}

\section{Introduction}

Glomus tumors are benign lesions that originate from modified smooth muscle cells of the glomus body that help regulate arteriolar blood flow. These tumors are commonly observed in the dermis or subcutis, but are rarely located in the stomach, occuring in $2 \%$ of all benign gastric tumors (1). Gastric glomus tumors are most commonly described as solitary, well-defined, submucosal lesions in the antrum, presenting with a variety of symptoms. Gastrointestinal bleeding with hematemesis/melena and epigastric discomfort are the most common initial symptoms and, in rare cases, may be life-threatening or lead to severe chronic anemia. Nausea and vomiting can also occur $(2,3)$. Surgery is often performed promptly since malignancy cannot be excluded due to the rarity of this tumor (4). Gastric glomus tumors have a good prognosis due to low recurrence and rarity of malignant transformation. However, a long follow-up is

Correspondence to: Professor Li Chen, Department of General Surgery, The Second Affiliated Hospital, College of Medicine, Zhejiang University, 88 Jiefang Road, Hangzhou, Zhejiang 310000, P.R. China

E-mail: chenli_hz@yahoo.com

Key words: gastric glomus tumor, immunohistochemistry, diagnosis, treatment required for further study. Patient provided written informed consent.

\section{Case report}

A 47-year-old female was admitted to The Second Affiliated Hospital of Zhejiang University (Hangzhou, China) on March 20, 2013 due to intermittent epigastralgia for four months, which could be temporarily relieved by eating. The patient denied any associated weight loss, fevers, chills, nausea, vomiting or melena. Acid suppression therapy had been administered with only minimal relief. A physical examination revealed only mild tenderness in the epigastric area, and serum levels of tumor makers were all within normal limits. The patient's father had died of gastric carcinoma.

Endoscopic ultrasound (EUS) revealed a protrusion on the posterior wall of the gastric antrum (Figs. 1 and 2A). Computed tomography (CT) scan identified a mass on the antrum $\sim 10 \mathrm{~mm}$ in diameter (Fig. 2B-D).

Immunoperoxidase stains revealed positive staining for smooth muscle actin (SMA) and collagen type IV, while being negative for synaptophysin, chromogranin A, laminin, S-100, cluster of differentiation (CD)34, CD31, CD99, cytokeratin (AE1/AE3), desmin and epithelial membrane antigen. The proliferation marker Ki-67 was positive in $<5 \%$ of tumor cell nuclei (Figs. 3-5).

A complete resection of the lesion was performed at The Second Affiliated Hospital of Zhejiang University. The patient was followed up for five months and recovered uneventfully without signs of relapse or gastrointestinal bleeding.

\section{Discussion}

Glomus tumors are rare in the stomach, and were first reported by De Busscher in 1948 as benign lesions (5). Gastric glomus tumors are now defined as mesenchymal tumors with potential malignant behavior. Malignant glomus tumors of the stomach with various organ metastases have been reported $(6,7)$. Criteria for identifying malignant potential in gastric glomus tumors remain to be established (8).

Pre-operative diagnosis of gastric glomus tumors is challenging and requires a multi-faculty medical approach. On unenhanced CT, they manifest as well-circumscribed submucosal masses with homogeneous density and may contain tiny 


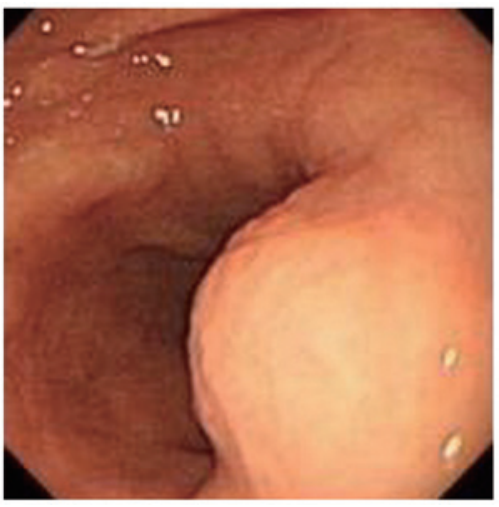

Figure 1. Endoscopy revealed a solid, elevated mass $20 \mathrm{~mm}$ in diameter.

flecks of calcification. Following the administration of contrast medium, these tumors demonstrate strong enhancement on arterial-phase scans and persistent enhancement on portal venous-phase scans (2). By contrast, the density of gastrointestinal stromal tumors is lower and these do not exhibit prolonged enhancement in the delayed phase (9). EUS features of gastric glomus tumors are heterogeneous, hypoechoic or hyperechoic, and hypervascular masses with internal hyperechoic spots and few tubular structures, mostly located on the fourth echolayer $(10,11)$. CT and EUS are useful in the early identification of gastric glomus tumors, particularly in terms of assessing tumor blood supply (12). On magnetic resonance images, gastric glomus tumors are marginally hypointense on T1-weighted images, slightly hyperintense on T2-weighted images, and hypervascular. In addition, gastric glomus tumors exhibit persistent enhancement following gadopentetate dimeglumine administration (13). Immunohistochemistry (IHC) is the preferred diagnostic tool, by which distinctive small, uniform and round tumor cells surrounding capillaries can be found, which are strongly positive for SMA, vimentin, calponin, collagen type IV and laminin (14).

Fine needle aspiration (FNA) can distinguish glomus tumors from more aggressive gastric tumors rapidly and pre-operatively, avoiding extensive surgical resection, particularly in larger tumors $(8,15,16)$. However, FNA can also incorrectly diagnose glomus tumors as leiomyomas or well-differentiated neuroendocrine tumors (2). Recently, Mohanty et al reported a case of gastric glomus tumor diagnosed by EUS-guided FNA and cell block IHC prior to endoscopic submucosal resection (ESMR) (17).

Complete surgical excision is the optimal treatment for a single lesion (18), although subtotal gastrectomy has been proposed for tumors suspected of malignancy (1). To minimize surgical trauma and the inflammatory response, the benign nature and small median size [varying between 2 and $3 \mathrm{~cm}$ (19)] of glomus tumors allows them to be removed by laparoscopic wedge resection (9,20-22), or endoscopic submucosal enucleation in select cases $(22,23)$ where the lesion is not close to the pylorus, porta hepatis and along the lesser curvature (24).

In summary, gastric glomus tumors are rare solitary submucosal tumors for which pre-operative diagnosis is challenging. Imaging findings assist significantly in making differential diagnoses. FNA with IHC may also be a promising method of diagnosis, helping the surgeon to plan an ESMR rather than more radical surgery. Exact diagnosis relies on histopathological examinations. Local resection by open or laparoscopic surgery is usually the most efficient therapy, although endoscopic submucosal enucleation may be another effective treatment.
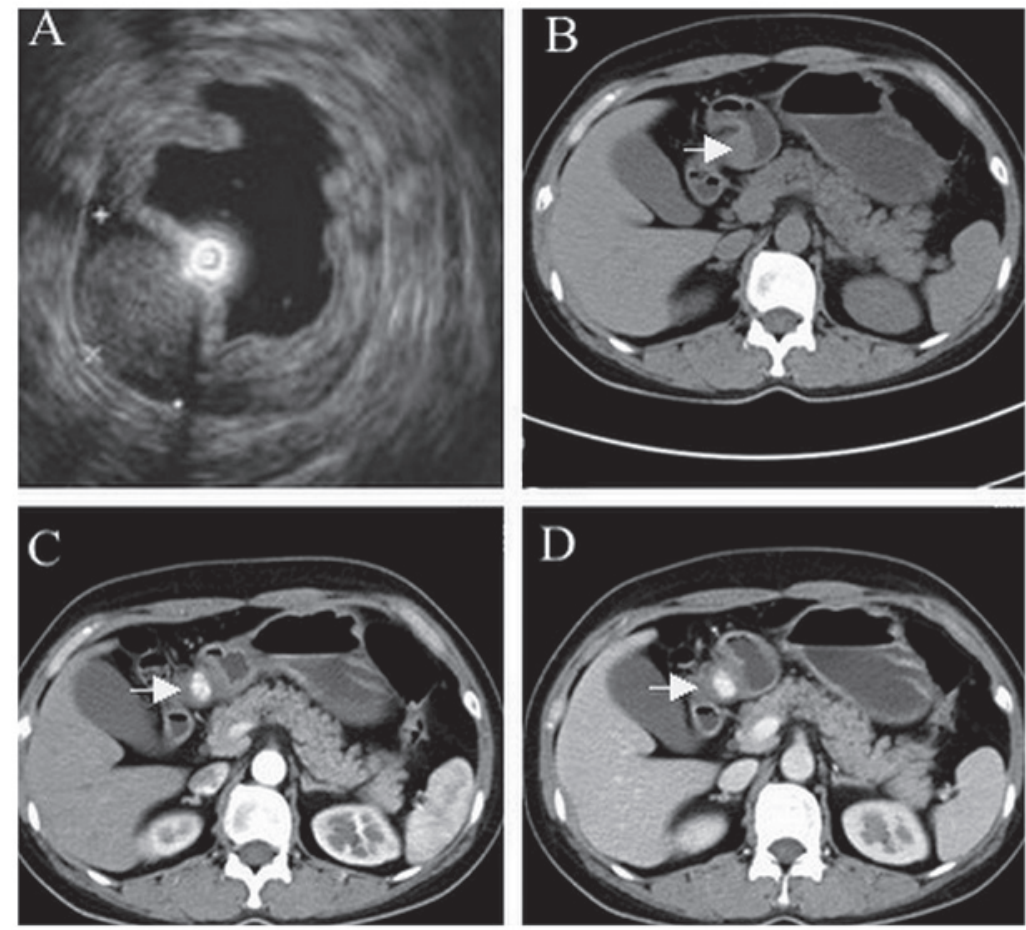

Figure 2. (A) EUS an ovoid, demarcated, heterogeneous, hyperechoic tumor 18.6x11.8 mm in size, originating from the fourth EUS layer (muscularis propria). Abdominal computed tomography revealed a well-demarcated, ovoid mass at the antrum (arrow) on (B) unenhanced, (C) arterial- and (D) delayed-phase scans. EUS, endoscopic ultrasound. 


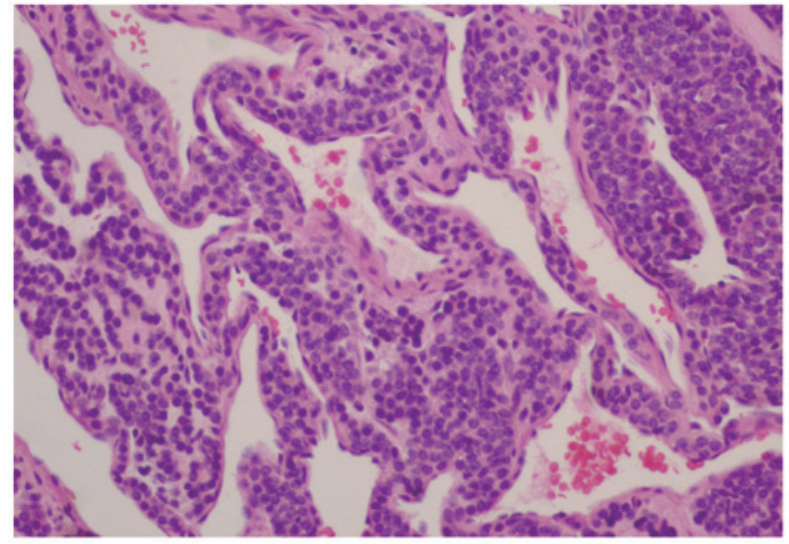

Figure 3. Microscopic examination revealed numerous dilated, thin-walled blood vessels, lined by a single layer of endothelial cells and surrounded by multilayer round glomus cells (hematoxylin and eosin staining; magnification, $\mathrm{x} 200)$.

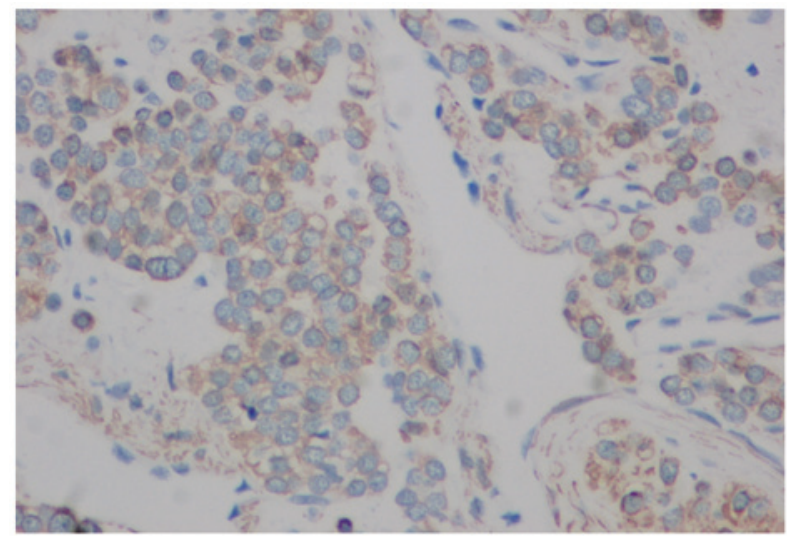

Figure 4. Tumor cells positive for smooth muscle actin, indicated by brown cytoplasmic stain (immunohistochemical staining; magnification, x200).

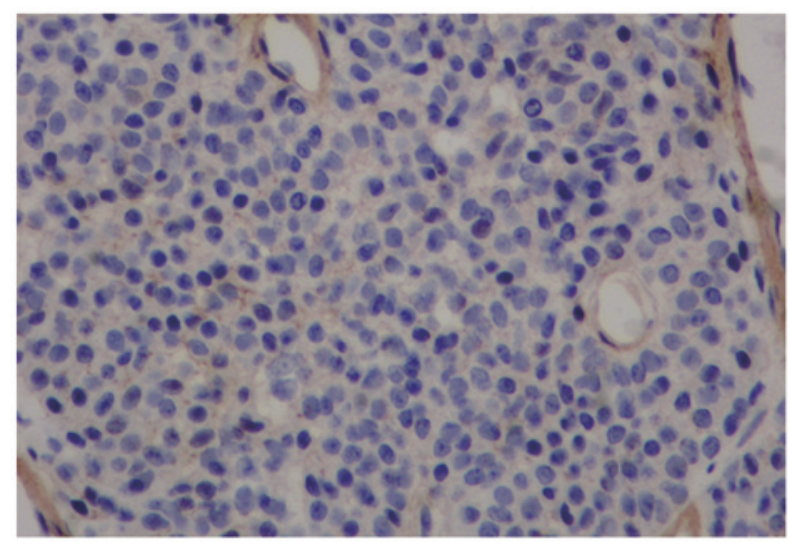

Figure 5. Tumor cells positive for collagen type IV (immunohistochemical staining; magnification, $\mathrm{x} 200$ ).

\section{Acknowledgements}

The authors are grateful to Jing-Hong $\mathrm{Xu}$, Department of Pathology, The Second Affiliated Hospital, College of Medicine, Zhejiang University, Hangzhou, China, for advice on histopathological examinations and helpful discussions.

\section{References}

1. Lee HW, Lee JJ, Yang DH and Lee BH: A clinicopathologic study of glomus tumor of the stomach. J Clin Gastroenterol 40: 717-720, 2006

2. Vassiliou I, Tympa A, Theodosopoulos T, et al: Gastric glomus tumor: a case report. World J Surg Oncol 8: 2010.

3. Nascimento EF, Fonte FP, Mendonça RL, Nonose R, de Souza CA and Martinez CA: Glomus tumor of the stomach: a rare cause of upper gastrointestinal bleeding. Case Rep Surg 2011: 371082, 2011.

4. Chou HP, Tiu CM, Chen JD and Chou YH: Glomus tumor in the stomach. Abdom Imaging 35: 390-392, 2010.

5. De Busscher G: Les anatomoses arterioveineuses de l'estomac: An ultrastructural study. Acta Neurol Morphol 6: 87-105, 1948.

6. Song SE, Lee CH, Kim KA, Lee HJ and Park CM: Malignant glomus tumor of the stomach with multiorgan metastases: report of a case. Surg Today 40: 662-667, 2010.

7. Bray AP, Wong NA and Narayan S: Cutaneous metastasis from gastric glomus tumour. Clin Exp Dermatol 34: e719-e721, 2009.

8. Huang CC, Yu FJ, Jan CM, et al: Gastric glomus tumor: a case report and review of the literature. Kaohsiung J Med Sci 26: 321-326, 2010.

9. Baek YH, Choi SR, Lee BE and Kim GH: Gastric glomus tumor: analysis of endosonographic characteristics and computed tomographic findings. Dig Endosc 25: 80-83, 2013.

10. Chou KC, Yang CW and Yen HH: Rare gastric glomus tumor causing upper gastrointestinal bleeding, with review of the endoscopic ultrasound features. Endoscopy 42: E58-E59, 2010.

11. Yan SL, Yeh YH, Chen CH, Yang CC, Kuo CL and Wu HS: Gastric glomus tumor: a hypervascular submucosal tumor on power Doppler endosonography. J Clin Ultrasound 35: 164-168, 2007.

12. Tang M, Hou J, Wu D, Han XY, Zeng MS and Yao XZ: Glomus tumor in the stomach: computed tomography and endoscopic ultrasound findings. World J Gastroenterol 19: 1327-1329, 2013.

13. Liu KL, Wang HP, Tseng WY, Shun CT, Chen SJ and Tsang YM: Glomus tumor of the stomach: MRI findings. AJR Am J Roentgenol 185: 1190-1192, 2005.

14. Fang HQ, Yang J, Zhang FF, Cui $Y$ and Han AJ: Clinicopathological features of gastric glomus tumor. World $\mathrm{J}$ Gastroenterol 16: 4616-4620, 2010.

15. Vinette-Leduc D and Yazdi HM: Fine-needle aspiration biopsy of a glomus tumor of the stomach. Diagn Cytopathol 24: 340-342, 2001.

16. Debol SM, Stanley MW, Mallery S, Sawinski E and Bardales RH: Glomus tumor of the stomach: cytologic diagnosis by endoscopic ultrasound-guided fine-needle aspiration. Diagn Cytopathol 28: 316-321, 2003

17. Mohanty SK, Pradhan D, Stavropoulos S, Donovan V and Gupta M: Diagnosis of gastric glomus tumour by endoscopic ultrasound-guided fine needle aspiration cytology: a case report. Cytopathology: May 1, 2013 (Epub ahead of print). doi: 10.1111/cyt.12068.

18. Kang G, Park HJ, Kim JY, et al: Glomus tumor of the stomach: a clinicopathologic analysis of 10 cases and review of the literature. Gut Liver 6: 52-57, 2012.

19. Miettinen M, Paal E, Lasota J and Sobin LH: Gastrointestinal glomus tumors: a clinicopathologic, immunohistochemical, and molecular genetic study of 32 cases. Am J Surg Pathol 26: 301-311, 2002.

20. Vanwijnsberghe S, Rubay R, Descamps C, Verdebout JM and Navez B: A glomic tumour of the stomach treated by laparoscopy. Acta Chir Belg 106: 613-615, 2006.

21. Campbell MJ, Irani S, Olgac S and Chang LC: Laparoscopic resection of a gastric glomus tumor. Indian J Surg 73: 230-232, 2011.

22. Zhang Y,Zhou P, Xu M, et al: Endoscopic diagnosis and treatment of gastric glomus tumors. Gastrointest Endosc 73: 371-375, 2011.

23. Xu M, Jiang XM, He YL, Zhang YL, Xu MD and Yao LQ: Glomus tumor of the stomach: A case treated by endoscopic submucosal dissection. Clin Res Hepatol Gastroenterol 35: 325-328, 2011.

24. Lorber J, Kalish J, Farraye FA, Cerda S and Babineau TJ: Glomus tumor of the gastric antrum: case report. Curr Surg 62: 436-438, 2005. 